\title{
Maintenance of head and neck tumor on- chip: gateway to personalized treatment?
}

\begin{abstract}
Aim: Head and neck squamous cell carcinomas (HNSCC) are solid tumors with low overall survival $(40-60 \%)$. In a move toward personalized medicine, maintenance of tumor biopsies in microfluidic tissue culture devices is being developed. Methodology/ results: HNSCC $(n=15)$ was dissected $(5-10 \mathrm{mg})$ and either analyzed immediately or cultured in a microfluidic device $\left(37^{\circ} \mathrm{C}\right)$ for $48 \mathrm{~h}$. No difference was observed in morphology between pre- and postculture specimens. Dissociated samples were analyzed using trypan blue exclusion (viability), propidium iodide flow cytometry (death) and MTS assay (proliferation) with no significant difference observed highlighting tissue maintenance. Computational fluid dynamics showed laminar flow within the system. Conclusion: The microfluidic culture system successfully maintained HNSCC for $48 \mathrm{~h}$, the culture system will allow testing of different treatment modalities with response monitoring.
\end{abstract}

Lay abstract: Head and neck cancers often have a poor treatment outcome. In order to study the response of the tissue, a miniaturized culture system has been developed to keep a small piece of tumor alive. In the current study, we show that small pieces of cancer tissue can be maintained in the system, using tissue structure and viability of single cells as a guide. In future work, patient equivalent treatments can be applied to these microculture systems to investigate individual patient tumor responses, which could help to guide treatment selection.

First draft submitted: 6 December 2016; Accepted for publication: 19 January 2017; Published online: 7 March 2017

Keywords: head and neck cancer $\bullet$ microfluidic $\bullet$ tissue culture $\bullet$ tumor

Head and neck cancers are a diverse group of malignancies arising from cells within the mucosal surfaces of the oral cavity, pharynx, larynx, salivary glands and nasal cavities [1] and are the sixth most common solid tumor worldwide [2] with squamous cell carcinomas (head and neck squamous cell carcinomas [HNSCC]) constituting 90\% [3]. A significant proportion of patients present with regional nodal involvement $(40 \%)$ and a further $10 \%$ present with the additional complication of distant metastasis [4]. Chemoradiation is the predominant primary treatment, but despite multiple advances over recent years, such as the introduction of intensity-modulated radiotherapy [5,6], treatment resistance is a persistent problem, contributing to the low overall 5-year survival figures of $40-60 \%$ for patients treated with radiotherapy or chemoradiotherapy [7].

As a result, the desire for targeted approaches to treatment has intensified $[8,9]$. However, to date cetuximab (the antiEGFR antibody) is the only approved targeted therapy for the treatment of recurrent metastatic head and neck disease [10]. Median duration of locoregional control was extended by 9.5 months with cetux-
Ruth Bower*,1, Victoria L Green', Elena Kuvshinova², Dmitriy Kuvshinov³, Laszlo Karsai $^{4}$, Stephen T Crank ${ }^{5}$, Nicholas D Stafford ${ }^{6}$ \& John Greenman ${ }^{1}$

'School of Life Sciences, The University of Hull, Cottingham Road, Hull, HU6 7RX, UK

${ }^{2}$ Department of Chemical \& Biological Engineering, The University of Sheffield, Mappin Street, Sheffield, S1 3JD, UK ${ }^{3}$ School of Engineering \& Computer Science, The University of Hull, Cottingham Road, Hull, HU6 7RX, UK ${ }^{4}$ Department of Cellular Pathology, Hull Royal Infirmary, Anlaby Road, Hull, HU3 2JZ, UK

${ }^{5}$ Department of Oral \& Maxillofacial Surgery, Hull Royal Infirmary, Anlaby Road, Hull, HU3 2JZ, UK

${ }^{6}$ Castle Hill Hospital, University of Hull, Daisy Building, Cottingham, HU16 5JQ, UK

*Author for correspondence:

R.Bower@2014.hull.ac.uk 
imab plus radiotherapy compared with radiotherapy alone [11]. In addition, searches for biomarkers to aid treatment selection have proved challenging with no standard-of-care biomarkers currently in use in all centers [12].

The human papilloma virus (HPV) is an important etiological agent in some subsets of HNSCC, with HPV-positive cancers having been shown to have a better prognosis and response to therapy than their HPV-negative counterparts [13,14]. HPV status is the strongest predictor of overall and disease-specific survival, as well as locoregional control [15]. Therefore, in a few centers, high-risk HPV variants are initially screened for positivity by p16 immunohistochemistry followed by PCR and/or in situ hybridization, in order to direct Phase III treatment de-escalation trials [4]. Beyond this, no technologies or screening methodologies to personalize therapy specifically to individual patients exist.

A large range of model systems exist to study tumor biology and treatment response including cell culture, spheroids, organoids and mouse models. While these methodologies have facilitated advancement of the field, they also have associated pitfalls; the use of a single cell type or even multiple cell types underestimates the complex nature of the in vivo microenvironment comprising numerous cell types in a unique spatial arrangement within an extracellular matrix [16]. Three-dimensional culture aims to recapitulate the spatial arrangement of cells yet still lacks complexity, including the full gamut of cell types such as tumorinfiltrating lymphocytes [17]. Xenograft models provide continuous flow to engrafted tumors within an in vivo environment, however, procedures are costly and lengthy and have clear ethical considerations [18], particularly at a time when there are calls for a reduction in the use of animals in research.

Microfluidic culture systems involve the continuous perfusion and removal of waste from a tissue sample $e x$ vivo, in a fashion mimicking the function of the circulatory system [19]. Multiple pieces from the same initial tissue sample can be perfused in parallel devices allowing the study of a range of variables with replicates. This provides a potential low-cost method for investigating the biology of patient samples and response to therapeutic assault in a personalized fashion.

Microfluidics has been defined as 'the science and technology of systems that process or manipulate small $\left(10^{-9}-10^{-18}\right.$ liters $)$ amounts of fluids, using channels with dimensions of tens to hundreds of micrometres' [20]. The small channel geometries afford the benefit of laminar flow within devices allowing a high level of spatial control. Laminar flow is defined at Reynolds numbers (Equation 1) below 2000 [21].
$\mathrm{Re}=\frac{\text { inertia force }}{\text { viscous force }}=\frac{\rho \mathrm{VD}}{\mu}$

Equation 1: Equation used to generate the Reynolds number within a microfluidic device, where $\rho(\mathrm{kg} /$ $\mathrm{m}^{3}$ ) is fluid density, $\mu$ is dynamic viscosity of fluid $(\mathrm{kg} / \mathrm{m} / \mathrm{s}), D_{\mathrm{h}}(\mathrm{m})$ is the hydraulic diameter and $V(\mathrm{~m} / \mathrm{s})$ is velocity.

Fluid flow modeling of devices by computational fluid dynamics (CFD) can afford benefits in the visualization of the properties discussed above, allowing manipulation of designs to achieve the closest possible resemblance of the in vivo environment for the biomedical model. CFD application in biomedical devices is relatively recent with more traditional applications being found in chemical and mechanical engineering [22].

The dawn of microfluidic applications in tissue culture began in 2003 with the culture of rat brain tissue slices [23], more recently (since 2013), various devices have been developed for the culture of human (Table 1) and animal tissue [24-28] with the potential of monitoring the response to therapeutic assault or external stimuli.

The capability of monitoring tissue response on an individual basis using microfluidic technology hopes to address the clinical problem of treatment resistance (Figure 1). The field has received some scepticism regarding the use of these devices for tissue maintenance with questions arising over sample viability and the relevance of the data obtained to the in vivo environment [29].

The aim of the current study was to address the uncertainty within this exciting field by generating sound evidence for the maintenance of malignant tissue within the microfluidic culture device, without loss of sample viability. Herein, flow cytometry is used to determine cell death within HNSCC samples before and after microfluidic culture; this is the first time these techniques have been used in conjunction to assess primary patient tissue. In addition, the in-house-designed microfluidic culture device was modeled for fluid flow dynamics.

\section{Materials \& methods}

\section{Patient biopsies}

Tumor tissue samples $(n=15)$ were obtained from patients with HNSCC in accordance with the Local Research Ethics Committee (LREC-10/H1304/6) and NHS R\&D approval (R0897), following written informed consent. Patients with no history of previous treatment undergoing resection surgery, to remove either laryngeal, oropharyngeal or oral cavity tumors staged at T2-T4 were recruited; associated metastatic lymph nodes staged at N1-N2 were also collected where possible. Fresh tissue samples were processed and cultured within 90 min of excision. 
Table 1. Published literature on the use of microfluidics for ex vivo tissue culture from human samples (1 January 2013 to 1 November 2016).

\begin{tabular}{|c|c|c|}
\hline Tissue & Summary of work conducted & Ref. \\
\hline $\begin{array}{l}\text { Intestinal sections (small } \\
\text { or large bowel) }\end{array}$ & $\begin{array}{l}\text { A dual-flow microfluidic device maintained full thickness intestinal tissue in known } \\
\text { orientation for up to } 3 \text { days. The device perfused luminal and serosal sides independently. } \\
\text { Tissue maintenance was demonstrated by LDH release, H\&E and Ki67 staining. ELISAs were } \\
\text { conducted on system effluents for Calprotein (s100a8; inflammatory marker) }\end{array}$ & [30] \\
\hline $\begin{array}{l}\text { Prostate and ovarian } \\
\text { cancer xenograft and } \\
\text { primary serous ovarian } \\
\text { cancer }\end{array}$ & $\begin{array}{l}\text { 'Spheroid-sized' MDTs ( } 380 \mu \mathrm{M} \text { diameter and } 300 \mu \mathrm{M} \text { height) were maintained in a } \\
\text { microfluidic device with regular medium replacement for up to } 8 \text { days. Viability was } \\
\text { determined on chip by confocal microscopy and off-chip by flow cytometry on dissociated } \\
\text { cells (annexin V and 7AAD, xenograft only). A proof of concept experiment showed } \\
\text { carboplatin chemosensitivity testing on a primary ovarian cancer tissue }\end{array}$ & [31] \\
\hline Human adipose tissue & $\begin{array}{l}\text { 3-mm diameter hAT biopsies maintained for up to } 7 \text { days were used to study insulin } \\
\text { resistance in Type } 2 \text { diabetes mellitus patients compared with healthy controls. Viability was } \\
\text { evaluated by MTT assay and morphology shown by off-chip H\&E staining. Glucose uptake } \\
\text { was used as a measure of insulin resistance }\end{array}$ & [32] \\
\hline $\begin{array}{l}\text { Prepuce and occipital } \\
\text { and temporal scalp skin } \\
\text { follicular unit extracts }\end{array}$ & $\begin{array}{l}\text { Culture device was developed to prolong culture period of skin biopsies and skin } \\
\text { equivalents. Punched biopsies ( } 5 \mathrm{~mm} \text { ) of prepuce and ex vivo FUEs were cultured } \\
\text { separately in the device for } 14 \text { days. Samples were taken from the device and cryosectioned } \\
\text { for downstream analysis of morphology (H\&E staining) and immunofluorescence for } \\
\text { proliferation (Ki67) and apoptosis (TUNEL) }\end{array}$ & [34] \\
\hline HNSCC & $\begin{array}{l}\text { HNSCC biopsy tissue ( } 5-10 \mathrm{mg} \text { ) maintained for up to } 9 \text { days was used to allow testing } \\
\text { of multiple chemotherapy agents (Cisplatin, Fluorouracil and Docetaxel) alone and in } \\
\text { combination. Response was measured in the system effluent; cell death was analyzed by } \\
\text { LDH and viability was measured by WST-1 proliferation assay }\end{array}$ & [35] \\
\hline \multicolumn{3}{|c|}{$\begin{array}{l}\text { 7AAD: 7-Aminoactinomycin D; FUE: Follicular unit extract; Gy: Gray; hAT: Human adipose tissue; H\&E: Hematoxylin and eosin; HNSCC: Head and neck squamous } \\
\text { cell carcinoma; LDH: Lactate dehydrogenase; MDT: Microdissected tissue; MF: Microfluidic; MTT: 3-(4,5-dimethylthiazol-2-yl)-2,5-diphenyltetrazolium bromide; } \\
\text { TUNEL: Terminal deoxynucleotidyl transferase dUTP nick-end labeling; WST: Water-soluble tetrazolium salt. }\end{array}$} \\
\hline
\end{tabular}

\section{Microfluidic device \& culture system}

The microfluidic device was fabricated from two layers of thermally bonded B270 glass in the Department of Chemistry at the University of Hull (Figure 2A), the upper layer was $3 \mathrm{~mm}$ in depth and lower glass layer $1 \mathrm{~mm}$. One inlet hole, one outlet hole and the central tissue chamber were drilled into the upper layer corresponding to channels $(170 \mu \mathrm{m}$ wide and $70 \mu \mathrm{m}$ deep) in the lower glass layer, fabricated by photolithography and wet etching techniques [36]. Over the central well, a PEEK plastic microport was bonded with epoxy adhesive, into which an English threaded adaptor (both IDEX Kinesis, Cambridgeshire, UK), filled with polydimethylsiloxane (Dow Corning, Barry, UK), was screwed to seal the chamber.

ETFE tubing (IDEX Kinesis, ) with an internal diameter of $0.5 \mathrm{~mm}$ was secured to the inlet and outlet hole by epoxy adhesive and a graphite ferrule (Figure 2A; IDEX Kinesis). Dulbecco's Modified Eagles Medium (DMEM; Lonza, Slough, UK) containing $25 \mathrm{mM}$ HEPES (4-(2-Hydroxyethyl) piperazine-1-ethanesulfonic acid) buffer and $4.5 \mathrm{~g} / \mathrm{l}$ glucose supplemented with $10 \%[\mathrm{v} / \mathrm{v}]$ fetal bovine serum (Labtech, East Sussex, UK), penicillin/streptomycin $0.1 \mathrm{U} / \mathrm{ml}$ and $0.1 \mathrm{mg} / \mathrm{ml}$, respectively, $0.1 \mathrm{mM} \mathrm{NEAA}$ (nonessential amino acids), $2.5 \mu \mathrm{g} / \mathrm{ml}$ Amphotericin B and $2 \mathrm{mM}$ L-glutamine (All Lonza; hereafter referred to as complete medium) was connected to the ETFE inlet tubing via a 2-part adapter (Lab smith, CA, USA). A 5-10-mg HNSCC sample was placed into the central tissue chamber and the system was connected to a Harvard PhD 2000 syringe pump (Harvard, UK), the device and tubing were maintained at $37^{\circ} \mathrm{C}$ inside an incubator with pressure-driven flow of complete medium at a volumetric flow rate of $2 \mu \mathrm{l} / \mathrm{min}$ (Figure 2B). Each device was run for $48 \mathrm{~h}$ with effluent collected in 1.5-ml Eppendorf tubes.

\section{Frozen tissue embedding \& morphological analyses}

Tumor sample was embedded in Tissue-Tek OCT (Optimum Cutting Temperature; Sakura, Berkshire, UK), by freezing in liquid nitrogen-cooled 2-methyl butane (Sigma-Aldrich, Dorset, UK) both prior to 
and following microfluidic culture. Tissue sections of $8 \mu \mathrm{M}$ were cut on a Leica CM1100 Cryostat and fixed for $20 \mathrm{~min}$ in $-20^{\circ} \mathrm{C}$ cooled methanol, stained in Harris' Hematoxylin (Sigma-Aldrich) for $5 \mathrm{~min}$ before differentiating in $1 \%$ acid alcohol (concentrated $\mathrm{HCl}$ in $70 \%$ Ethanol) and rinsing in running tap water. The tissue was then stained with $1 \%(\mathrm{w} / \mathrm{v}$ ) Eosin Y (SigmaAldrich) in tap water for 5 min before washing in tap water. The tissue was dehydrated through graded alcohols (70, 90 and 100\%) and three changes of histoclear before mounting in histomount. Slides were visualized using light microscopy (Nikon ECLIPSE E800) and photographed (Micropublisher 5.0 Real Time Viewing) utilizing Image-Pro premier software (MediaCybernetics, Cambridge, UK). Detailed observations of the tissue characteristics were carried out under close supervision of a consultant head and neck pathologist, Lazslo Karsai.

\section{Tissue dissociation}

Dissociation of tumor tissue prior to and following microfluidic culture was achieved using a combination of mechanical and enzymatic methods; samples of a known weight were placed into a secured lid of a $1.5-\mathrm{ml}$ Eppendorf tube under aseptic conditions and minced using a criss-cross cutting action. Complete medium $(1 \mathrm{ml})$ containing $0.02 \%(\mathrm{w} / \mathrm{v}$ ) Collagenase IV (SigmaAldrich) and $0.02 \%$ (w/v) DNase I (Roche, Hertfordshire, UK) was added to the Eppendorf tube and the lid containing the minced tissue was replaced before incubation on a rotator for $2 \mathrm{~h}$ at $37^{\circ} \mathrm{C}$ and $5 \% \mathrm{CO}_{2}$. Following enzyme incubation, the suspension was strained (70- $\mu \mathrm{m}$ cell strainer, BD, Oxford, UK) and centrifuged at $400 \times \mathrm{g}$ for $5 \mathrm{~min}$, the pellet was resuspended in $1 \mathrm{ml}$ of complete medium and percentage viability of tissue determined using Trypan Blue exclusion.

\section{Propidium iodide flow cytometry}

The resultant cell suspension from the dispersal of a single 5-10-mg tissue sample $(\mathrm{n}=8)$ prior to and after microfluidic culture was washed in phosphate-buffered saline (PBS; $5 \mathrm{ml}$ ) containing $0.25 \%(\mathrm{w} / \mathrm{v}) \mathrm{BSA}$ (Bovine Serum Albumin; Sigma-Aldrich) and 0.06\% (w/v) sodium azide (Sigma-Aldrich; PBS/BSA/Azide) and divided between two tubes before centrifugation at $400 \times g$ for $5 \mathrm{~min}$. Pellets were resuspended in 100$\mu \mathrm{l} \mathrm{PBS} / \mathrm{BSA} /$ Azide, and either stained with 500- $\mu \mathrm{g} /$ $\mathrm{ml}$ propidium iodide (PI; Sigma-Aldrich) for $15 \mathrm{~min}$ in the dark and or remained unstained as a control.

Following a further wash, the cells were resuspended in 300- $\mu \mathrm{l} \mathrm{PBS/BSA/Azide} \mathrm{and} \mathrm{analyzed} \mathrm{in} \mathrm{the} \mathrm{FL-3} \mathrm{chan-}$ nel on a flow cytometer (BD FACSCalibur), a minimum of 10,000 events were acquired and a histogram was plotted to determine the percentage of dead cells.

\section{MTS proliferation assay}

Metabolic activity/proliferation of dissociated cells prior to and following microfluidic culture was determined using the Promega CellTiter 96 ${ }^{\circledR}$ AQueous One Solution (Promega, Southampton, UK) according to the manufacturer's instructions. Briefly, dissociated cells from a single 5-10-mg tissue were resuspended in $200 \mu \mathrm{l}$ of complete DMEM. The suspensions were cultured in duplicate overnight in a 96-well flat-bottomed plate before the MTS assay reagent $(20 \mu \mathrm{l})$ was added to each well for $2 \mathrm{~h}$ at $37^{\circ} \mathrm{C}$ prior to measuring absorbance of the formazan product at $492 \mathrm{~nm}$ (Thermo Scientific Multiskan FC, Thermo Scientific, Hampsted, UK). Absorbance values were standardized per milligram of starting weight of tissue.

\section{Statistics}

Statistical analyses were performed using SPSS statistics 23 (IBM, Portsmouth, UK) and p-values $<0.05$ were considered significant. Differences between the preculture and postculture samples were tested for significance using independent samples' t-tests.

\section{Fluid flow modeling}

Generally, fluid dynamics is described by governing equations [37]:

Conservation of mass equation:

$$
\nabla \cdot u=0
$$

where $u$ is flow velocity vector.

Conservation of momentum equation for incompressible fluid:

$$
\rho \frac{d u}{d t}=\nabla \cdot \sigma
$$

where $\rho$ is density, $t$ is time, $\sigma$ is total stress;

$\sigma=-p I+2 \eta D$

where $p$ is pressure, $\eta$ is dynamic viscosity, $D$ is rate of strain tensor, $\boldsymbol{I}$ is identical matrix:

$$
\mathrm{D}=\frac{1}{2}\left[\nabla u+(\nabla u)^{\mathrm{T}}\right]
$$

where ${ }^{T}$ shows the transpose operation.

Computer simulation of pressure-driven Poiseuille flow in the chip was realized using COMSOL software, version 5.2a (COMSOL, Cambridge, UK). The discretization of governing partial differential equations was made by the finite element method. Rheological (flow) parameters of the DMEM medium were assumed to be the same as water (temperature = $37^{\circ} \mathrm{C}$ ). The meshing was performed using the phys- 


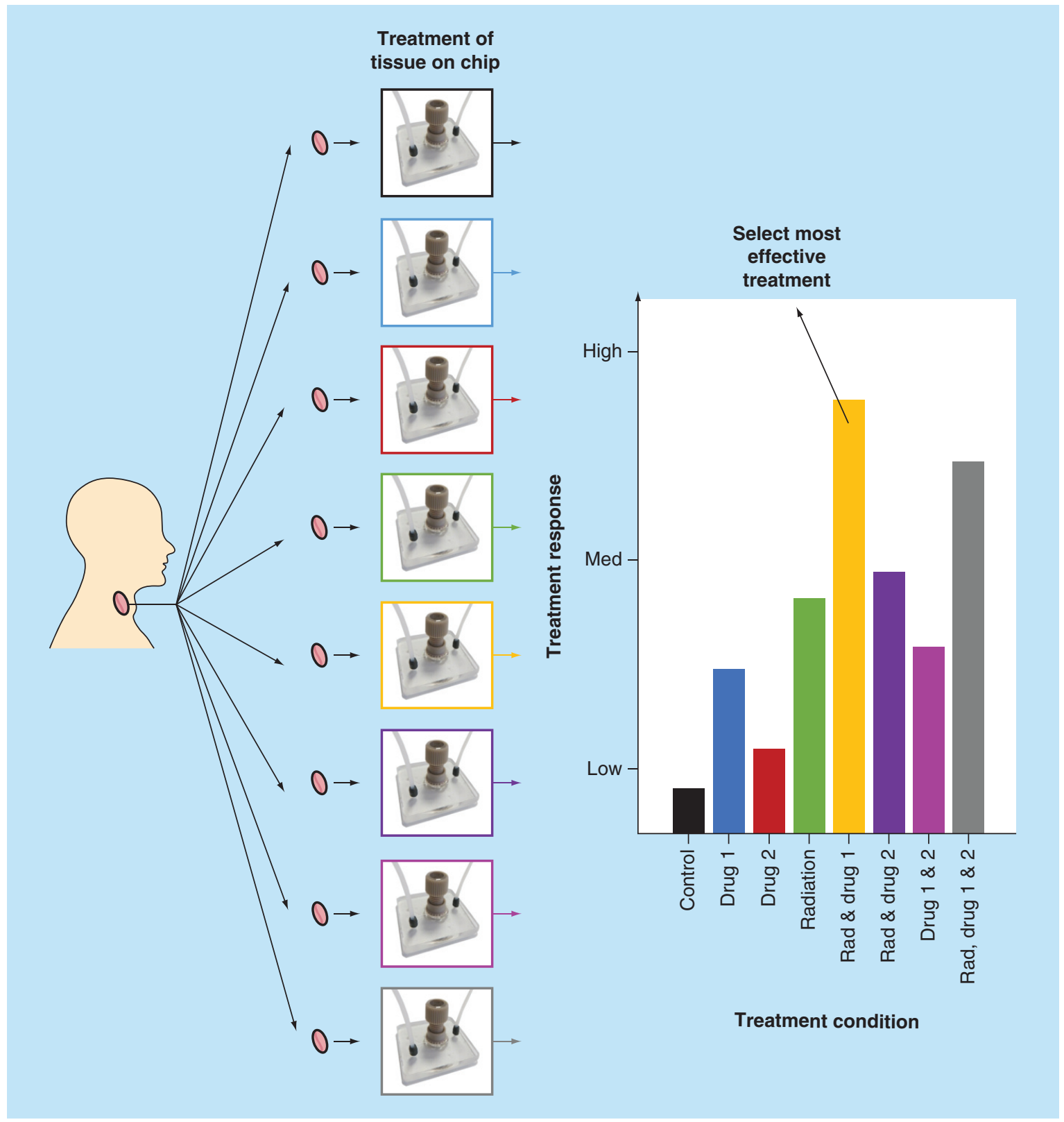

Figure 1. Schematic diagram showing the parallel nature of the on-chip treatment testing concept. Ex vivo testing of tissue response provides potential for the selection of treatment on an individual patient basis.

ics-controlled method with normal-sized triangle elements from 0.003 to $0.1 \mathrm{~mm}$, the total number of elements was 14,736 . The inlet flow rate was set as an inlet boundary condition, and the volumetric flow rate of $2 \mu \mathrm{l} / \mathrm{min}$ was transformed with regard to the inlet width of $0.5 \mathrm{~mm}$. At the outlet, $\mathrm{p}=0$ is established.

Navier-Stokes governing equations for incompressible steady-state flow real geometry of the chip were solved using COMSOL Multiphysics modelling software to obtain velocity field and flow pattern for Newtonian fluid. The built-in 'Laminar flow' module for Newtonian fluid (constant dynamic viscosity) was selected to simulate fluid flow at given conditions.

\section{Results}

Tumor characteristics of patient biopsies, tumor site, $\mathrm{T}$ and $\mathrm{N}$ classification and patient age and gender were collected ( $n=15$; Supplementary Table 1).

\section{Morphological analyses}

Hematoxylin and eosin (H\&E)-stained HNSCC tissue sections were visualized before placement of the tumor in the microfluidic culture device (Figure 3, left panel) and following $48 \mathrm{~h}$ of continuous perfusion within the device (Figure 3, right panel).

Representative images are shown from two patients with laryngeal squamous cell carcinoma (A \& B). The 

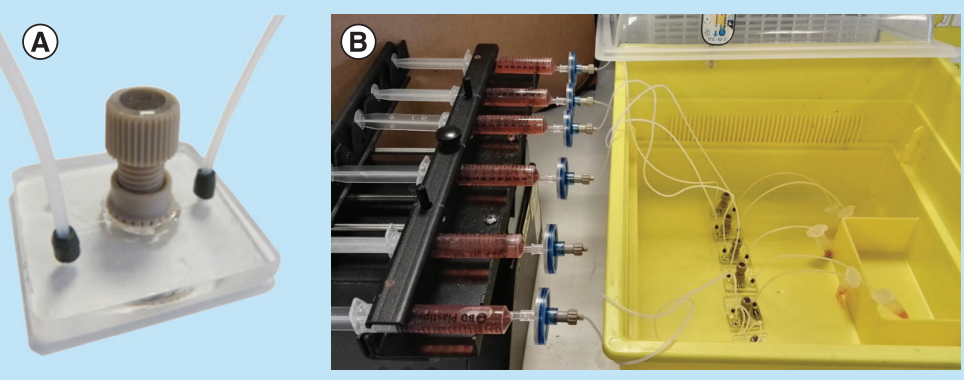

Figure 2. Photographs of microfluidic culture device and setup. (A) Device close up including PEEK plastic microport and PDMS-filled PEEK-threaded adaptor. Connected to inlet and outlet ETFE tubing via graphite ferrules. (B) Complete culture setup with syringes containing complete medium attached to a pressure-driven syringe pump for continuous flow to the tissue sample within the device. Effluent was collected in Eppendorf tubes and the devices were maintained in an incubator at $37^{\circ} \mathrm{C}$.

ETFE: Ethylene tetrafluoroethylene; PDMS: Polydimethylsiloxane; PEEK: Polyether ether ketone.

low-magnification $(100 \times)$ images show maintenance of the tissue structure. Infiltrating islands and interconnected cords of tumor cells are shown in the premicrofluidic culture images and are retained following 48-h culture. Pale pink stromal areas of the tumor are also apparent before and after microfluidic incubation.

The higher magnification $(400 \times)$ allows the assessment of cellular characteristics and shows an intact basement membrane following microfluidic culture. Furthermore, the orientation of cell maturation seen in premicrofluidic culture tissue, with more mature cells observed in the center of tumor islands and nests was also present post-48-h microfluidic culture. The chronic inflammatory mononuclear cell fraction (lymphocytes and macrophages) within the stroma was recognizable both before and after microfluidic culture.

\section{Viability analyses}

No significant difference in percentage viability between tumor tissue obtained following surgical resection (11-57.9\%) and a sample from the same tumor cultured in the microfluidic device (17.1$40.4 \%)$ for $48 \mathrm{~h}$ was observed using trypan blue exclusion (Figure $4 \mathrm{~A} ; \mathrm{n}=15$ ). The viable number of cells obtained per milligram of tissue was also calculated, with tissue dissociated immediately yielding an average of $5.71 \times 10^{4} \pm 3.14 \times 10^{4}$ viable cells $/ \mathrm{mg}$ tissue, and that dissociated following $48 \mathrm{~h}$ of microfluidic culture yielding $4.36 \times 10^{4} \pm 1.63 \times 10^{4}$ viable cells $/ \mathrm{mg}$ tissue, again with no significant difference between the two conditions. For comparison, flow cytometry was utilized to quantify cell death by PI staining; again no significant difference in percentage cell death was observed between the dissociated tumor cells before and after 48-h microfluidic culture (Figure 4B; $\mathrm{n}=8$ ). The viability (by trypan blue) and cell death (as measured by PI staining) results were concordant.

\section{Tumor cell proliferation}

A variation in proliferative capacity of the dissociated tumor cells was observed between samples with formazan product absorbance $(492 \mathrm{~nm})$ values ranging from 0.026 to 0.140 in preculture samples and that from post-48-h culture samples being 0.022-0.164 (per milligram of tissue). Consequently, no significant difference in proliferation of the cell suspensions was observed between pre- and post-48-h culture (Figure 4C; $n=7$ ).

\section{Device modeling}

The experimental cell configuration is not axisymmetric, but symmetric along the central plane. This plane, which crosses the input-output channel along the middle line, is the most information-rich area, and provides the flow pattern, characterized by highest reynolds number variability. Thus, based on the assumption, that further developing of the model to $3 \mathrm{D}$ would not contribute significantly to the flow profile investigation, and would greatly increase simulation time, the decision was made to apply the $2 \mathrm{D}$ model to the study. This model allows investigation of the main issue of the study: the flow uniformity.

In the first instance, the 'perfect' flow simulation was modeled that is where the placement of the tissue sample within the device was central with no deformation to the shape of the tissue apparent as a result of the force exerted by the flow (Figure 5).

As the structure, shape and the placement of the sample within the chamber of the device could all be changed as a result of the flow forces acting on the tissue. The device was modeled with a tilt of 5 and $10^{\circ}$ to show the change to the fluid flow in this situation (Figure 6; $10^{\circ}$ model).

In order to quantify the flow characteristics, numerically five points around the tissue were chosen for fur- 
ther study (Figure 7A). Point 3 was placed $0.1 \mathrm{~mm}$ away from the tissue boundary while points 1, 2, 4 and 5 were placed $0.25 \mathrm{~mm}$ away from the tissue boundary in all tilt conditions (except in the case of point 4 in the $10^{\circ}$ tilt analysis where the distance was $0.09 \mathrm{~mm}$ ). Calculated values for Reynolds number and velocity magnitude are shown (Table 2).

The Reynolds numbers obtained show values' characteristic of laminar flow at the 0,5 and $10^{\circ}$ tilt conditions. Velocity magnitude data shows key differences within the fluid flow at point 4 in the tilted $10^{\circ}$ condition, given the reduced distance between the tissue and the chamber wall the velocity magnitude was increased by over $2.5 \times$ compared with the $0^{\circ}$ initial, no tilt condition. Furthermore, the velocity magnitude at point 4 was $5.3 \times$ higher than that at point 2 in the $10^{\circ}$ tilt condition, whereas in the $0^{\circ}$ tilt condition point 4 velocity was only $1.26 \times$ higher than point 2 . Differences in velocity at all other points were minimal.

\section{Discussion}

The biological data yielded promising results in terms of viability maintenance following microfluidic culture; intact morphology following microfluidic culture was demonstrated by $\mathrm{H} \& \mathrm{E}$ staining with the presence of distinct characteristics of squamous cell carcinoma. This is in agreement with previous studies from the group, using both rat liver and HNSCC tissue $[38,39]$ and those of others who used H\&E to demonstrate maintenance of tissue-specific structures including single hair follicular units and human adipose tissue $[32,34]$ with culture times in microfluidic devices ranging from 1 to 14 days.

The use of enzymatic and mechanical tissue dissociation to obtain a single cell suspension for subsequent analysis allows a more precise determination of cell viability and increases the scope of analytical methods that are achievable; however, it may also increase the chance of experimental-induced damage. The results obtained from single cell suspensions showed concordance between trypan blue exclusion and PI flow cytometry. Previously, the use of flow cytometry following microfluidic culture has received little attention and has only been utilized on prostate and ovarian cancer xenograft tissues [31]. In that study, the maintenance of small microdissected tissues (MDTs; $380 \mu \mathrm{M}$ diameter and $300 \mu \mathrm{M}$ height) was maintained in a microfluidic device with periodic media changes, continuous perfusion was not employed. MDTs were stained with annexin $\mathrm{V}$ and 7AAD prior to dissociation $(0.25 \mathrm{mg} /$ $\mathrm{ml}$ collagenase IV in saline $15 \mathrm{~min}$ at $37^{\circ} \mathrm{C}$ ) followed by flow cytometric analysis. A minimum of $40 \%$ viability (shown by unstained cells) for 8 days across four different xenografts was obtained. Results presented in the current study, using PI to assess cell death, detail the first flow cytometry data on human primary tissue following microfluidic culture, and showed no increase in cell death following the 48-h culture period, although the data reveal lower viabilities (preculture average: $28.9 \%$, post- 48 -h culture average: $28.1 \%$ ) than the $40 \%$ reported previously [31]. This could be attributable to the difference in starting material (xenograft vs primary tissue) and the experimental procedure differences, particularly staining the tissue with flow cytometry markers before or after dissociation. Other work maintaining HNSCC in microfluidic devices showed no significant difference in apoptotic cell death (apoptotic index, m30 immunohistochemistry [IHC] staining) between preculture and post-4-day culture, again indicating maintenance of tissue [33]; however, samples were not disaggregated for cellular analysis.

The range of viable cells/mg obtained experimentally from HNSCC tumors before $\left(1.5 \times 10^{4}-1.4\right.$

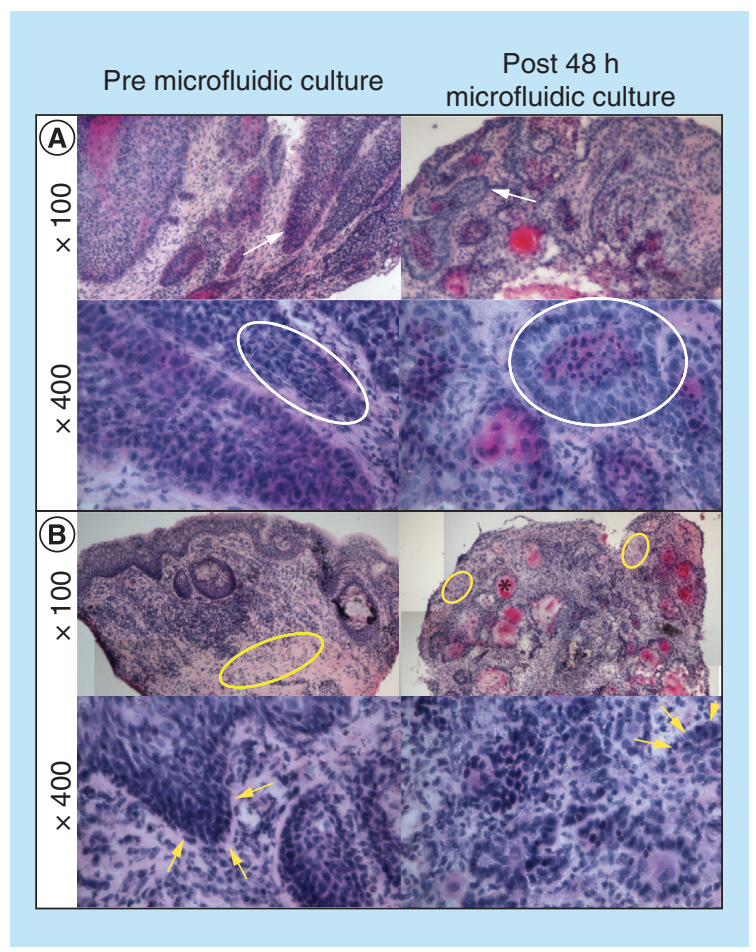

Figure 3. Hematoxylin and Eosin-stained 8- $\mu \mathrm{m}$ tissue sections, shown at $100 \times$ and $400 \times$ magnification. Two representative tumors are shown $(n=15)$. (A) Laryngeal squamous cell carcinoma, staging T4aNOMO. (B)

Transglottic laryngeal squamous cell carcinoma, staging T4N0M0. Each tumor is shown pre- and post-48-h microfluidic culture. The bright pink-stained areas on some of the images depict extracellular keratin (keratin pearls*), arising from the squamous cells. White arrows show infiltrating islands and cords of tumor cells. Tumor area showing orientation of cell maturation is circled in white. Pale stromal areas with inflammatory cells are circled in yellow. Intact basement membrane is shown by yellow arrows. 
(A)

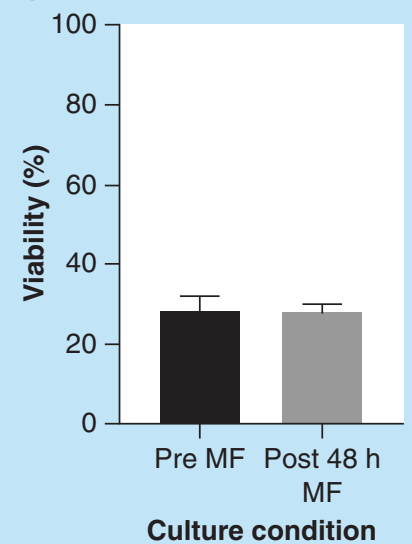

(B)

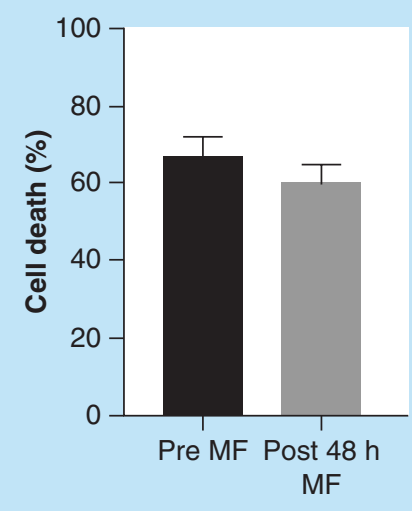

Culture condition
(C)

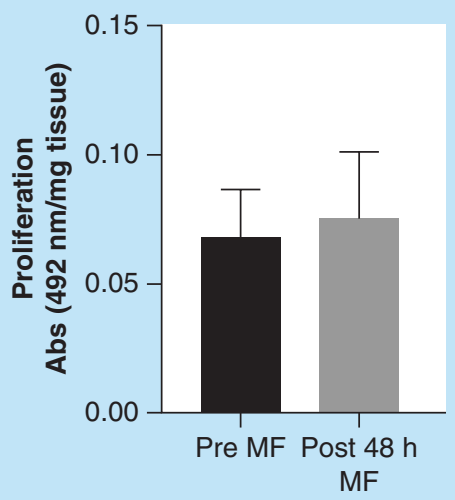

Culture condition

Figure 4. Viability, cell death and proliferation analysis of single cell suspensions from dispersed tumor tissue before and after microfluidic culture. (A) Percentage viability of cell population by trypan blue exclusion $(n=15)$. (B) Percentage cell death by PI staining and flow cytometry from 10,000 events $(n=8)$. (C) Proliferation (MTS assay) of cell suspensions following overnight culture quantified by absorbance of colored formazan product at $492 \mathrm{~nm}$ and normalized per milligram of tissue $(n=7)$. Mean + SEM.

$\mathrm{PI}$ : Propidium iodide.

$\left.\times 10^{5}\right)$ and after 48-h microfluidic culture (1.9-7.6 $\times$ $\left.10^{4}\right)$ demonstrated no significant effect of microfluidic culture on the number of viable cells within the tumor tissue. Trypan blue exclusion has previously been used following combined mechanical and enzymatic dissociation methods (tissue minced, incubated $4^{\circ} \mathrm{C}$ with $0.5 \%$ trypsin $12-20 \mathrm{~h}$, then agitated with rotating razor blades for 5-15 $\mathrm{min}$ ) on solid tumors' (small-cell lung carcinomas and testicular cancer) preculture yielding $1.5-5 \times 10^{4}$ viable cells $/ \mathrm{mg}[40]$, thus our experimental system appears to extend the range from that previously reported.

The current study showed no significant difference between the average proliferation of HNSCC samples preculture and post-48-h microfluidic culture by the MTS proliferation assay, as the variance in the results observed was high (0.026-0.146 and 0.022-0.164, respectively, absorbance ${ }_{492 \mathrm{~nm}} / \mathrm{mg}$ tissue). The range in proliferation is a relevant observation from the data and correlates with the known intertumor heterogeneity affecting key cancer pathways driving phenotypic variation [41]. Proliferation of HNSCC is known to vary between anatomical subsites, for example, Pedicini et al., correlated EGFR expression of patient tumors to HNSCC subsites with high-EGFR expression associated with greater proliferation following irradiation [42]. Further variation is also seen within tumors, (intraheterogeneity: $[41,43,44])$ which could in part explain the variation seen between the preculture and post-48-h microfluidic culture conditions. The use of other metabolic activity assays, similar to MTS, as a surrogate for proliferation has been used by

Table 2. Velocity magnitude of fluid flow around the sample with different displacement angles $(0$, 5 and $10^{\circ}$ ) and Reynolds number within the device.

\begin{tabular}{|c|c|c|c|c|c|}
\hline \multirow{2}{*}{$\begin{array}{l}\text { Angle of tilt } \\
\text { (degrees) }\end{array}$} & \multicolumn{5}{|c|}{ Velocity magnitude $(\mathrm{mm} / \mathrm{s})$} \\
\hline & Point 1 & Point 2 & Point 3 & Point 4 & Point 5 \\
\hline 0 & 0.2 & 0.19 & 0.04 & 0.24 & 0.24 \\
\hline 5 & 0.19 & 0.17 & 0.039 & 0.26 & 0.26 \\
\hline 10 & 0.16 & 0.12 & 0.039 & 0.64 & 0.3 \\
\hline \multicolumn{6}{|c|}{ Reynolds number } \\
\hline 0 & $1.9 \times 10^{-3}$ & $1.3 \times 10^{-3}$ & $4.4 \times 10^{-4}$ & $1.5 \times 10^{-3}$ & $2.2 \times 10^{-3}$ \\
\hline 5 & $9.4 \times 10^{-4}$ & $6.0 \times 10^{-4}$ & $1.4 \times 10^{-4}$ & $1.0 \times 10^{-3}$ & $1.4 \times 10^{-3}$ \\
\hline 10 & $1.6 \times 10^{-3}$ & $1.3 \times 10^{-3}$ & $3.4 \times 10^{-4}$ & $5.1 \times 10^{-3}$ & $3.1 \times 10^{-3}$ \\
\hline
\end{tabular}


others (MTT assay); to compare human adipose tissue before and after 7 days of culture in a microfluidic device again with no difference in absorbance observed between the preculture and following microfluidic culture conditions [32].

Maintenance of patient tissue on a chip is an emerging technology with cell culture or 'engineered tissue' culture on a chip more widely employed; in part due to the relative simplicity of sample acquisition and availability. However, the use of cell lines, spheroids and engineered tissue will always simplify the clinical situation and as such often extend culture periods. For example, spheroids created from a breast cancer cell line or a breast cancer cell line coculture with a fibroblast cell line were maintained for up to 14 days shown using Calcein AM (AM; acetoxymethyl ester group) as a viability marker [48]. Utilizing primary tissue samples as an alternative holds key benefits in the translational relevance and application of the data obtained from the devices to real-world problems, such as treatment resistance.

The use of modeling to better understand the flow patterns within microfluidic devices can aid under- standing and interpretation of experimental observations and results; this extra dimension provides information regarding the surrounding environment of the tissue sample. Furthermore, the modeling can be used as a tool to improve the design of devices in order to enhance the functionality of the device and/or mimic the in vivo situation. The Reynolds number obtained for the device in the current study indicates that the flow within the channels is 'creeping flow' also known as the Stokes law region (Reynolds number $<1[49]$ ), where the viscous forces of the fluid dominate over the inertial forces, due to the low fluid velocities [50]. Thus, the flow within the device can be classified as laminar, a key property in the employment of microfluidic devices in biomedical applications.

Although this laminar flow in the current device has been utilized successfully to maintain HNSCC tissue, the modeling has identified some areas for improvement, namely the stagnant flow areas around the base of the tissue within the chamber; these areas result in reduced perfusion of the tissue. Moreover, it could not be excluded that at higher mass inflow the sample would

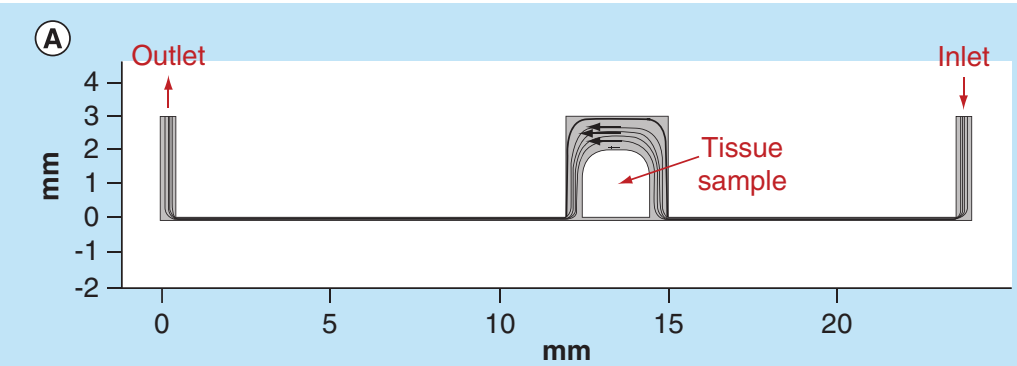

(B)

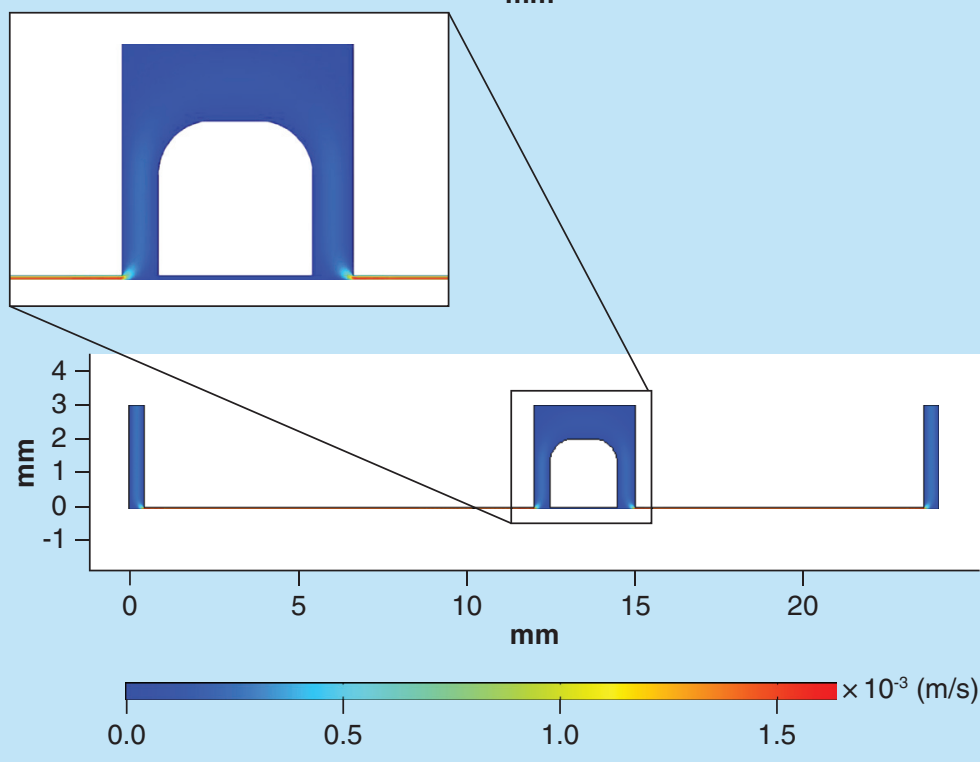

Figure 5. Computer simulation of fluid flow and velocity field in the microfluidic culture device. (A) Streamlines and velocity vectors of laminar fluid flow in the device. Labels show inlet, outlet and tissue sample location. (B) Velocity magnitude of the flow within the device. Color legend explains value of the velocity along the channel and the treatment chamber $(\mathrm{m} / \mathrm{s})$; thus, the maximum of the velocity equals $1.5 \times 10^{-3} \mathrm{~m} / \mathrm{s}$ or $1.5 \mathrm{~mm} / \mathrm{s}$. 
not touch the chamber walls due to flow pressure, so this positioning of the tissue would form a turbulent flow pattern with vorticities and zones with velocities close to zero. Device modeling has been utilized previously to develop designs that allow manipulation of different experimental conditions; for example, mathematical modeling described fluid dynamics alongside intracellular signaling as a key predictor in glucose uptake in adipose tissue [32] and assessment of oxygen delivery to tumor samples within a microfluidic device [31].

\section{Conclusion}

Microfluidic culture successfully maintains HNSCC samples for $48 \mathrm{~h}$ such that they are comparable to the preculture sample in terms of morphology and viability with expected variation between patients. The study provides scope for extending this time period in future work. The current microfluidic device design facilitated laminar flow, however, the design also resulted in some 'dead zones' where the fluid velocity was virtually nonexistent resulting in limited perfusion. Despite this, the maintenance of the tissue viability within the microfluidic device is encouraging and invites subsequent studies applying treatment regimens to HNSCC samples within optimized on-chip devices.

\section{Future perspective}

Treatment resistance is a persistent problem yielding a worse prognosis and reducing clinical success for patients [51]. The selection of a treatment for an individual patient based on the responsiveness of their malignancy to therapeutic assault could customize care and improve outcomes. Culture of small HNSCC samples using microfluidic culture as described or in fact the culture of any solid tumor could facilitate such testing, including that of novel agents. Additionally, modifications in device design guided by CFD could improve perfusion to the whole surface of the tissue and as such increase the length of the culture period. Future work on treatment application to these devices will increase the translational relevance of the technology allowing monitoring of patient-specific tumors and their response to therapeutic assault, in order to direct treatment decision-making.
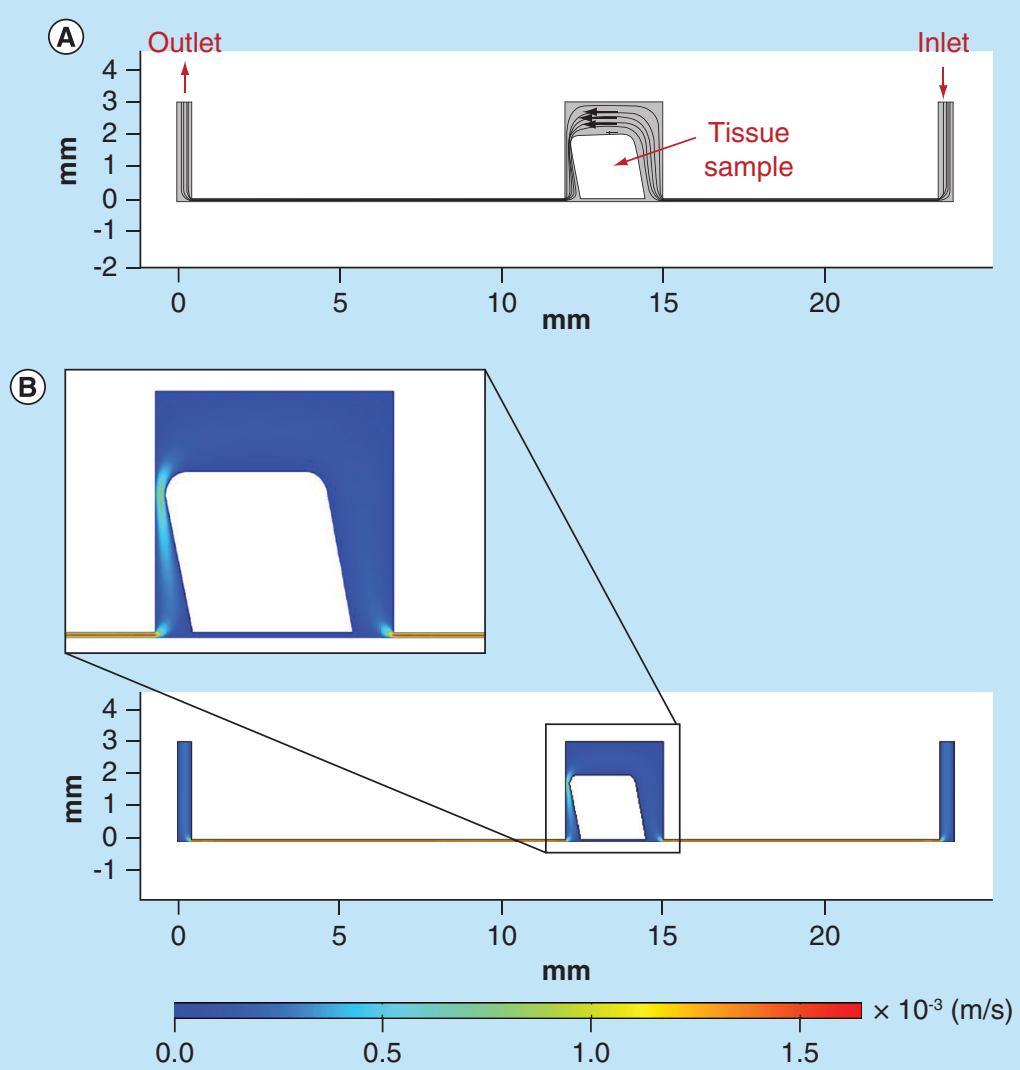

Figure 6. Computer simulation of fluid flow in the microfluidic tissue culture device, with tissue sample tilted $10^{\circ}$ due to the flow pressure. (A) Streamlines and velocity vectors of laminar fluid flow in the device. Labels show inlet, outlet and tissue sample location. (B) Velocity magnitude of the flow within the device. Color legend explains value of the velocity along the channel and the treatment chamber $(\mathrm{m} / \mathrm{s})$; thus, the maximum of the velocity equals $1.5 \times 10^{-3} \mathrm{~m} / \mathrm{s}$ or $1.5 \mathrm{~mm} / \mathrm{s}$. 


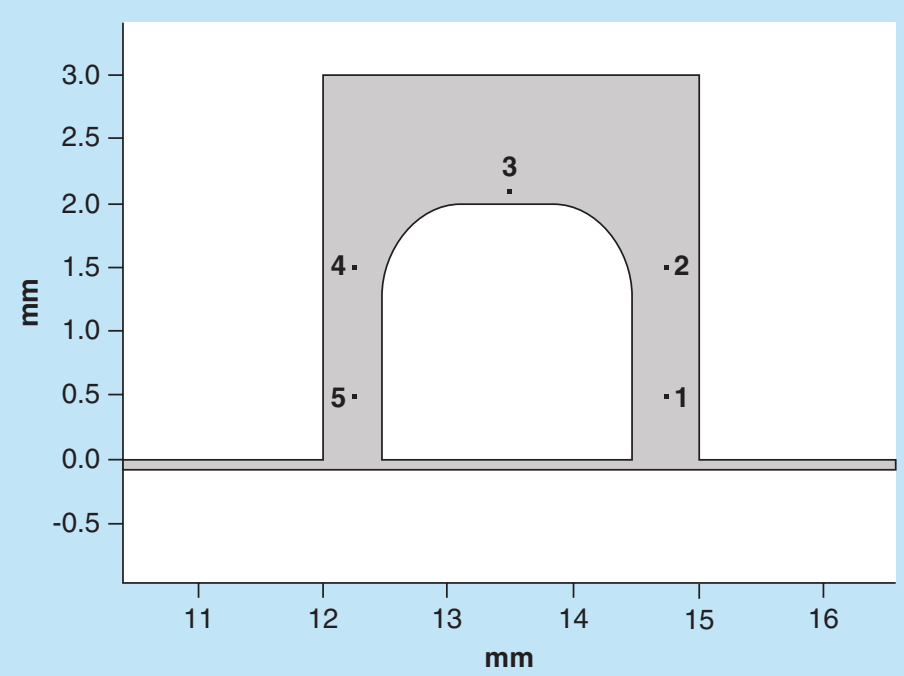

Figure 7. Comparative analysis of the velocity magnitude and Reynolds number of the flow along the sample. Point placement along the sample for further analysis.

\section{Supplementary data}

To view the supplementary data that accompany this paper please visit the journal website at: www.future-science/doi/ full/10.2217/fsoa-2016-0089

\section{Author contributions}

R Bower, V Green and J Greenman were involved in the design of the experiments, interpretation of the data and writing the article. E Kuvshinova, D Kuvshinov and R Bower were involved in computational fluid dynamics, interpretation of the output and writing the relevant sections. L Karsai reviewed the histology sections and was involved in the analysis of these data. S Crank and N Stafford collected HNSCC specimens. All authors approved the final version of the manuscript.

\section{Acknowledgements}

The authors would like to acknowledge the patients of Hull and East Yorkshire NHS Trust who allowed samples to be used in this research and the assistance of Jemy Jose alongside the
ENT and Oral \& Maxillofacial theatre teams for the sample collection. R Bower received a PhD studentship from the University of Hull Brignall Scholarship, without this the work herein would not have been possible.

\section{Financial \& competing interests disclosure}

The authors have no relevant affiliations or financial involvement with any organization or entity with a financial interest in or financial conflict with the subject matter or materials discussed in the manuscript. This includes employment, consultancies, honoraria, stock ownership or options, expert testimony, grants or patents received or pending, or royalties.

No writing assistance was utilized in the production of this manuscript.

\section{Open access}

This work is licensed under the Creative Commons Attribution 4.0 License. To view a copy of this license, visit http://creativecommons.org/licenses/by/4.0/

\section{Executive summary}

- Head and Neck Squamous Cell Carcinomascan be maintained in our tumor-on-chip device without loss of sample viability for $48 \mathrm{~h}$.

- Squamous cell carcinoma tissue morphology characteristics are maintained following culture.

- Computational fluid dynamics alongside biological data is valuable in terms of characterizing the sample environment and improving the model system.

- Establishment of an optimized, defined, model system will allow tissue to be tested for response to different treatment modalities simultaneously.

\section{References}

Papers of special note have been highlighted as: $\bullet$ of interest;

•• of considerable interest

1 McMahon S, Chen AY. Head and neck cancer. Cancer Metastasis Rev. 22, 21-24 (2003).
2 Louie KS, Mehanna H, Sasieni P. Trends in head and neck cancers in England from 1995 to 2011 and projections up to 2025. Oral Oncol. 51(4), 341-418 (2015).

- Outlines the burden and outlook of head and neck squamous cell carcinoma in the UK. 
3 Dobrossy L. Epidemiology of head and neck cancer: magnitude of the problem. Cancer Metastasis Rev. 24(1), 9-17 (2005).

4 Marur S, Forastiere AA, Torre LA et al. Head and neck squamous cell carcinoma: update on epidemiology, diagnosis, and treatment. Mayo Clin. Proc. 91(3), 386-396 (2016).

5 De Neve W, De Gersem W, Madani I. Rational use of intensity-modulated radiation therapy: the importance of clinical outcome. Semin. Radiat. Oncol. 22(1), 40-49 (2012).

6 Ghosh-Laskar S, Yathiraj PH, Dutta D et al. Prospective randomized controlled trial to compare 3-dimensional conformal radiotherapy to intensity-modulated radiotherapy in head and neck squamous cell carcinoma: long-term results. Head Neck. 38(S1), E1481-E1487 (2016).

7 Perri F, Pacelli R, Della Vittoria Scarpati G et al. Radioresistance in head and neck squamous cell carcinoma: biological bases and therapeutic implications. Head Neck. 37(5), 763-770 (2015).

8 Akervall J, Nandalur S, Zhang J et al. A novel panel of biomarkers predicts radioresistance in patients with squamous cell carcinoma of the head and neck. Eur. J. Cancer 50 (3), 570-581 (2014).

9 Suh Y, Amelio I, Guerrero Urbano T, Tavassoli M. Clinical update on cancer: molecular oncology of head and neck cancer. Cell Death Dis. 5(1), e1018 (2014).

10 Echarri M, Lopez-Martin A, Hitt R. Targeted therapy in locally advanced and recurrent/metastatic head and neck squamous cell carcinoma (LA-R/M HNSCC). Cancers (Basel). 8(3), 27 (2016).

11 Bonner JA, Harari PM, Giralt J et al. Radiotherapy plus cetuximab for squamous-cell carcinoma of the head and neck. N. Engl. J. Med. 354(6), 567-578 (2006).

12 Morris LGT, Chandramohan R, West $\mathrm{L}$ et al. The molecular landscape of recurrent and metastatic head and neck cancers. JAMA Oncol. 46(2), 114-117 (2016).

13 Lassen P, Eriksen JG, Krogdahl A et al. The influence of HPV-associated p16-expression on accelerated fractionated radiotherapy in head and neck cancer: evaluation of the randomised DAHANCA 6\&7 trial. Radiother. Oncol. 100(1), 49-55 (2011).

14 Ziemann F, Arenz A, Preising S et al. Increased sensitivity of HPV-positive head and neck cancer cell lines to $x$-irradiation \pm Cisplatin due to decreased expression of E6 and E7 oncoproteins and enhanced apoptosis. Am. J. Cancer Res. 5(3), 1017-1031 (2015).

15 Friedman JM, Stavas MJ, Cmelak AJ. Clinical and scientific impact of human papillomavirus on head and neck cancer. World J. Clin. Oncol. 5(4), 781-791 (2014).

16 Hickman JA, Graeser R, de Hoogt R et al. Threedimensional models of cancer for pharmacology and cancer cell biology: capturing tumor complexity in vitro/ex vivo. Biotechnol. J. 9(9), 1115-1128 (2014).

17 Kim S-A, Lee EK, Kuh H-J. Co-culture of 3D tumor spheroids with fibroblasts as a model for epithelialmesenchymal transition in vitro. Exp. Cell Res. 335(2), 187-196 (2015).
18 Stebbing J, Paz K, Schwartz GK et al. Patient-derived xenografts for individualized care in advanced sarcoma. Cancer 120(13), 2006-2015 (2014).

19 Dawson A, Green V, Bower R, Greenman J. Microfluidics: towards personalised medicine in cancer therapy. Drug Target Rev. 3, 12-17 (2016).

- Review of the use of microfluidic tissue culture devices in cancer research.

20 Whitesides GM. The origins and the future of microfluidics. Nature 442(7101), 368-373 (2006).

- Landmark paper on the science behind and the use of microfluidic technology in multiple applications.

21 Avila K, Moxey D, de Lozar A, Avila M, Barkley D, Hof B. The onset of turbulence in pipe flow. Science 333(6039), 192-196 (2011).

22 Huang M, Fan S, Xing W, Liu C. Microfluidic cell culture system studies and computational fluid dynamics. Math. Comput. Model. 52(11), 2036-2042 (2010).

23 Passeraub PA, Almeida AC, Thakor NV. Design, microfabrication and analysis of a microfluidic chamber for the perfusion of brain tissue slices. Biomed. Microdevices. 5(2), 147-155 (2003).

24 Komeya M, Kimura H, Nakamura $\mathrm{H}$ et al. Long-term ex vivo maintenance of testis tissues producing fertile sperm in a microfluidic device. Sci. Rep. 6, 21472 (2016).

25 Dodson KH, Echevarria FD, Li D, Sappington RM, Edd JF. Retina-on-a-chip: a microfluidic platform for point access signaling studies. Biomed. Microdevices. 17(6), 114 (2015).

26 Yasotharan S, Pinto S, Sled JG, Bolz S-S, Günther A. Arteryon-a-chip platform for automated, multimodal assessment of cerebral blood vessel structure and function. Lab Chip 15(12), 2660-2669 (2015).

27 Chang TC, Mikheev AM, Huynh W, Monnat RJ, Rostomily RC, Folch A. Parallel microfluidic chemosensitivity testing on individual slice cultures. Lab Chip 14 (23), 4540-4551 (2014).

28 Silva PN, Green BJ, Altamentova SM, Rocheleau JV. A microfluidic device designed to induce media flow throughout pancreatic islets while limiting shear-induced damage. Lab Chip 13(22), 4374-4384 (2013).

29 van Midwoud PM, Merema MT, Verpoorte E, Groothuis GMM. Microfluidics enables small-scale tissue-based drug metabolism studies with scarce human tissue. J. Lab. Autom. 16(6), 468-476 (2011).

30 Dawson A, Dyer C, Macfie J et al. A microfluidic chip based model for the study of full thickness human intestinal tissue using dual flow. Biomicrofluidics 10(6), 64101 (2016).

31 Astolfi M, Péant B, Lateef MA et al. Micro-dissected tumor tissues on chip: an ex vivo method for drug testing and personalized therapy. Lab Chip 16(2), 312-325 (2015).

-. A tissue on a chip device developed with computer modeling for chemotherapy testing with the use of flow cytometry to monitor tissue viability.

32 Zambon A, Zoso A, Gagliano O et al. High temporal resolution detection of patient-specific glucose uptake from 
human ex vivo adipose tissue on-chip. Anal. Chem. 87(13), 6535-6543 (2015).

Dreen VL, Stafford ND, Greenman J. Analysis of radiation-induced cell death in head and neck squamous cell carcinoma and rat liver maintained in microfluidic devices. Otolaryngol. Head Neck Surg. 150(1), 73-80 (2014).

34 Ataç B, Wagner I, Horland R et al. Skin and hair on-a-chip: in vitro skin models versus ex vivo tissue maintenance with dynamic perfusion. Lab Chip 13(18), 3555 (2013).

35 Sylvester DC, Hattersley SM, Stafford ND, Haswell SJ, Greenman J. Development of microfluidic-based analytical methodology for studying the effects of chemotherapy agents on cancer tissue. Curr. Anal. Chem. 9(1), 2-8 (2013).

36 McCreedy T. Rapid prototyping of glass and PDMS microstructures for micro total analytical systems and micro chemical reactors by microfabrication in the general laboratory. Anal. Chim. Acta 427(1), 39-43 (2001).

Morrison FA. An Introduction to Fluid Mechanics. Cambridge University Press, UK (2013).

38 Hattersley SM, Dyer CE, Greenman J, Haswell SJ. Development of a microfluidic device for the maintenance and interrogation of viable tissue biopsies. Lab Chip 8(11), 1842-1846 (2008).

39 Hattersley SM, Sylvester DC, Dyer CE, Stafford ND, Haswell SJ, Greenman J. A microfluidic system for testing the responses of head and neck squamous cell carcinoma tissue biopsies to treatment with chemotherapy drugs. Ann. Biomed. Eng. 40(6), 1277-1288 (2012).

40 Engelholm SA, Spang-Thomsen M, Brünner N, Nøhr I, Vindeløv LL. Disaggregation of human solid tumours by combined mechanical and enzymatic methods. Br. J. Cancer 51(1), 93-98 (1985).

41 Burrell RA, McGranahan N, Bartek J, Swanton C. The causes and consequences of genetic heterogeneity in cancer evolution. Nature 501(7467), 338-345 (2013).
42 Pedicini P, Nappi A, Strigari L et al. Correlation between EGFR expression and accelerated proliferation during radiotherapy of head and neck squamous cell carcinoma. Radiat. Oncol. 7(1), 143 (2012).

43 Ledgerwood LG, Kumar D, Eterovic AK et al. The degree of intratumor mutational heterogeneity varies by primary tumor sub-site. Oncotarget 7(19), 27185-27198 (2016).

44 Rocco JW. Mutant Allele Tumor Heterogeneity (MATH) and head and neck squamous cell carcinoma. Head Neck Pathol. 9(1), 1-5 (2015).

45 Ma C, Zhao L, Zhou E-M, Xu J, Shen S, Wang J. Onchip construction of liver lobule-like microtissue and its application for adverse drug reaction assay. Anal. Chem. 88(3), 1719-1727 (2016).

46 Kim HJ, Ingber DE. Gut-on-a-chip microenvironment induces human intestinal cells to undergo villus differentiation. Integr. Biol. (Camb). 5(9), 1130-1140 (2013).

47 Ruppen J, Wildhaber FD, Strub C et al. Towards personalized medicine: chemosensitivity assays of patient lung cancer cell spheroids in a perfused microfluidic platform. Lab Chip 15(14), 3076-3085 (2015).

48 Sabhachandani P, Motwani V, Cohen N, Sarkar S, Torchilin V, Konry T. Generation and functional assessment of 3D multicellular spheroids in droplet based microfluidics platform. Lab Chip 16(3), 497-505 (2016).

49 Smith R. Chemical Process Design and Integration. Wiley, Chichester, UK (2014).

50 Brody JP, Yager P, Goldstein RE, Austin RH. Biotechnology at low Reynolds numbers. Biophys. J. 71, 3430-3441 (1996).

51 Brana I, Siu LL. Locally advanced head and neck squamous cell cancer: treatment choice based on risk factors and optimizing drug prescription. Ann. Oncol. 23(Suppl. 10), 178-185 (2012). 